\section{The Third Order Nonlinear Optical Properties of Gold Nanoparticles in Glasses, Part I}

\author{
Daven Compton, Lesley Cornish, Elma van \\ der Lingen
}

Mintek, Private Bag X3015, Randburg, 2125, South Africa

Email: Davenc@mintek.co.za

\begin{abstract}
The optical properties of gold nanoparticles have been known for a number of years and recent advances in laser power have now allowed the non-linear optical properties to be studied. In this short review paper, the various theories that have been used to describe the optical properties of gold nanoparticles are presented. Methods of preparing gold nanoparticles in glasses are explained briefly, as well as characterization techniques. The optical properties of gold nanoparticles are reviewed, as well as the effects of particle size, shape, concentration and glass type.
\end{abstract}

\section{Introduction}

In the search for new materials, nanoparticle research offers great potential. Size confinement results in changes in the electronic properties of the nanoparticles, and thus the optical properties. This may possibly suit many electronic and opto-electronic applications.

Materials with third-order nonlinearity are essential for light-controlled phase and refractive index modulation for future applications in optical computing, real time holography, optical correlators, and phase conjugators $(1,2,3)$. Glasses and metal colloids are being investigated in the expectation that their large third-order optical non-linear susceptibility $\left(\chi^{(3)}\right)$ will be used as optical logic devices in the optical information field. Glasses with gold nanoparticles (2-30 nm in size) have been extensively investigated, since they have high optical nonlinearity, fast response time (in the order of a femtosecond) and a suitable operating wavelength (corresponds to the second harmonic generation of a $\mathrm{Nd}$ :YAG laser). The non-linear index of refraction is also proportional to the $\chi^{(3)}$, and this is very important in optical switching devices and waveguides.

To design optical materials, suitable constituent materials must be found and characterised, and relationships predicting the effective optical parameters must be determined. In glass composites, the glass is nonabsorbing throughout the visible spectrum while the metal is highly absorbing and reflective throughout the same range. Yet the composite displays a resonance absorption peak within the visible, giving its characteristic colour. It is important to study the relationship between particle radius, matrix and nanoparticles interaction, $\chi^{(3)}$ and the absorption coefficient.

To stabilise and incorporate nanoparticles into solid matrices for devices requires an understanding of the interactions between the matrix and the nanoparticles, and their influence on the whole system. In general, the growth of Au nanoparticles in glasses leads to an increase in $\chi^{(3)}$ and concurrently an increase in the absorption coefficient. The maximum of $\chi^{(3)}$ occurs at the same wavelength where the optical density has a peak value. For practical applications, it is desirable to have a large figure of merit. A typical figure of merit for third-order nonlinearities is given by

$$
F=\chi^{(3)} / \alpha \tau
$$

where $\alpha$ is the linear absorption coefficient and $\tau$ is the response time. It can be seen that even if $\chi^{(3)}$ is large, the improvement in the figure of merit may not be large due to linear absorption $(\alpha)$, which is enhanced at resonance. It is also seen that the figure of merit has a time factor $(\tau)$. Materials with a fast response time are highly desirable. 


\section{Theories of the Optical Properties of Nanoparticles}

The basic underlying theory used to describe nanoparticles is mature and there have been a number of modifications to this theory. As this report is a brief review, only the important and pertinent theories are mentioned. Even so, the details of the mentioned theories have been left out, as there are excellent reviews by Gehr and Boyd (4) and Boyd et al. (5), which cover the mathematical aspects very well. The interested reader is directed to these publications for detailed study. A general overview of optical modelling and the properties of metal nanoparticles can be found in the text by Feldheim and Foss (6). They discuss many aspects of metal nanoparticles, including second-order non-linear effects, which are not covered in this review.

To describe a nano-composite material, the nanoparticle dimensions and spacing must be smaller than the radiation wavelength. The nano-composite can then be described by effective optical parameters that are related to the constituent parameters. When the spacing between the nanoparticles is comparable to the wavelength, effective medium theory cannot describe the properties well. With this in mind, when metal nanoparticles (with diameters much less than the wavelength of the pumping radiation) are embedded in a glass matrix, the optical properties can be described using effective medium theory for small noninteracting particles in a dielectric (7). Even though effective medium theory predicts that metal nanoparticles smaller than the wavelength of the radiation are transparent $(8,9)$, when incorporated in a dielectric matrix, the optical absorption is expected to show a peak at the surface plasmon resonance (SPR) frequency. The SPR frequency is dependent on both the electronic properties of the nanoparticles, and the index of refraction of the matrix.

When the underlying geometry of nanoparticles in a matrix is unknown, it has been shown (10) that it is not possible to predict the effective medium parameters. However, it is possible to determine upper and lower limits of the effective linear dielectric constant. The simplest limits are the Wiener limits (11), but it is possible to find more restrictive limits such as Hashin and Shtrikman (12) and Bergman (13). When certain geometries are considered, then solutions to the effective medium theory can be obtained, and the values of the effective medium parameters can be determined.

\section{Maxwell-Garnett (MG) Theory}

Maxwell-Garnett $(14,15)$ developed one of the earliest effective medium theories, which was very successful in accurately predicting the location of the SPR in gold-doped glass through the effective dielectric constant. The theory assumes the nanoparticles are spherical, uniform in size and smaller than the interparticle distance, which in turn is much smaller than the wavelength. For the assumptions to be valid, the volume fraction of the nanoparticles must be less than $\sim 10 \%$. The derivation is valid in general, in that the nanoparticles need not be metals, but another dielectric as well.

By considering the nanoparticles as point dipoles (ignoring their size), then the following equation can be derived from Maxwell's equations:

$$
\frac{\mathcal{E}_{\text {effective }}-\boldsymbol{\varepsilon}_{\text {matrix }}}{\mathcal{E}_{\text {effective }}+2 \mathcal{E}_{\text {matrix }}}=\beta f \text { where } \beta \equiv \frac{\mathcal{E}_{\text {particle }}-\boldsymbol{\varepsilon}_{\text {matrix }}}{\mathcal{E}_{\text {particle }}+2 \mathcal{E}_{\text {matrix }}},
$$

where $f$ is the volume fill fraction of the nanoparticles. Close inspection of the equation shows that if the nanoparticles have a negative real part of the dielectric constant $\left(\boldsymbol{\mathcal { E }}_{\text {particle }}\right.$ is $\mathrm{Re}(-\mathrm{ve}))$, then there exists a resonance. This resonance will depend on the dielectric constant of the matrix and the nanoparticle. The above holds for spherical shapes, but Cohen et al. (16) have generalized the theory to take nanoparticles with ellipsoid shapes into account.

Mie developed a theory (17) to explain how particle size gave rise to various colours of colloidal gold solutions (18). The theory is limited to nanoparticles with spherical geometry and nanoparticle separation distances greater than the wavelength of the radiation, but is valid for particles of varying size. The theory predicts experimental results well, and further predicts that off the SPR frequency, the absorption should increase with decreasing wavelength. This theory is the simplest consideration of effective medium theory.

Another concept from MG theory is that of the local field. The applied electric field from the incident radiation is not the same electric field that polarizes the nanoparticle. The local field is an effective field that polarizes the individual nanoparticle, and its value depends on the polarization of the matrix, as well as the applied electric field. The local field factor provides a measure of how the average electric field in a given component of the composite is related to the spatially averaged electric field. The local field factor of the local field enhancement strongly depends on the refractive index and dielectric constant of the matrix. Therefore the choice of the matrix is a very important determinant of the non-linear optical properties. These local field effects are significant in non-linear effective medium theories, as the correction factor for the local field appears multiple times in the expressions for the non-linear susceptibilities. The MG theory has been extended into the non-linear regime (19). It was found that:

$\delta \boldsymbol{\varepsilon}_{\text {effective }}=f\left(\frac{3 \boldsymbol{\varepsilon}_{\text {matrix }}}{\boldsymbol{\varepsilon}_{\text {particle }}+2 \boldsymbol{E}_{\text {matrix }}}\right)^{2}\left|\frac{3 \boldsymbol{\varepsilon}_{\text {matrix }}}{\boldsymbol{\varepsilon}_{\text {particle }}+2 \boldsymbol{E}_{\text {matrix }}}\right|^{2} 12 \pi \chi^{(3)}{ }_{\text {particle }}|\mathrm{E}|^{2}$. 
In the expression, the local field correction factor (the terms in parentheses) appears to the fourth power, and exhibits the same resonance condition as before. The possibility of strong resonant enhancement of the thirdorder susceptibility $\left(\chi^{(3)}\right.$ - the susceptibility is basically just a measure of how easily a material can be polarised) is seen. MG theory predicts a resonance (SPR), and that at this resonance, the third-order non-linear susceptibility $\left(\chi^{(3)}\right)$ will be enhanced. It must also be remembered that the absorption increases at this resonance as well.

Agarwal and Dutta Gupta (20) used another formulation for the non-linear optical properties of composites in the MG model. They arrived at a more accurate description, although the difference in the low fill fraction limit is very small. Sipe and Boyd (21) developed their own non-linear theory based on the MG model. For non-linear nanoparticles in a linear host, they found no enhancement of $\chi^{(3)}$ was possible through the local field correction factors. In the case of linear nanoparticles in a non-linear matrix, they found enhancement possible when the particles have a much larger linear index of refraction than the matrix. The reason given was that the dipole field of the nanoparticles forces the electric field to concentrate in the matrix, greatly increasing the local field there. Their theory also considered the case when both constituents of the composite were non-linear, the result being the sum of the results for the two cases of one linear and the one non-linear. An interesting prediction of their theory is that $\chi^{(3)}$ will become greater the larger the fill fraction.

Stroud and Hui (22) derived an integral expression for the effective medium, arriving at the same relation as Ricard et al. (19). Zeng et al. (23) performed a Taylor expansion of the linear effective dielectric constant, and arrived at a more accurate answer for the effective nonlinearity when the inclusions are non-linear.

The MG model in the non-linear regime has been generalised by Haus et al. (24), who have performed calculations for ellipsoidal shaped nanoparticles. In another report, Haus et al. (25) found that the particle shape could control the position of the resonance.

Another modification to the MG model is particles consisting of a spherical core surrounded by a spherical shell. Neeves and Birnboim $(26,27)$ determined their effective third-order susceptibilities for both electrorestrictive and electronic contributions. Using the metallic core to increase the field in the shell (or vice versa), they found it is possible to achieve a resonant enhancement of $\chi^{(3)}$ and a corresponding increase in the figure of merit, since the absorption does not increase as rapidly as the nonlinearity near resonance. Zhang and Stroud (28) considered particles consisting of a gold core with an unspecified non-linear material for a shell. They found that enhancements on the order of 100 were possible. They also commented that there was an optimal shell thickness, depending on the parameters of the materials used. Zhou et al. (29) made gold coatings on the surface of goldsulphide nanoparticles, and found good agreement with theoretical predictions. Kalyaniwalla et al. (30) extended the analysis to the case of confocal ellipsoids, specifically considering the case of a semiconductor core surrounded by a metal shell.

García et al. (31) modified the MG model to incorporate two parameters to account for geometrical and distribution homogeneity effects in a simple manner, avoiding the complications of scattering (large particles) and quantum effects (very small particles). Thus, the modified model is restricted to small clusters (gold colloids in the $\sim 6$ to $50 \mathrm{~nm}$ diameter) at low concentrations, and is valid for nonscattering particles in the so-called quasi-static approximation. Good agreement was found with experimental results in the case of Au-colloids embedded into silica or alumina coatings by the sol-gel method.

Hache et al. (32) considered theoretically the various mechanisms contributing to the nonlinearity of the optical properties of small gold particles. They suggested three possible origins of the nonlinearity of the optical properties of metal particles: intraband (due to electron transitions within a single conduction band $-\chi^{(3)}$ is negative imaginary, scaling as the inverse of the radius cubed); interband (electron transitions from the $d$-band to the s-conduction-band $-\chi^{(3)}$ is negative imaginary and independent of size); and hot-electron contributions (due to non equilibrium heating of the conduction band electrons $-\chi^{(3)}$ positive imaginary and independent of size). They found the hot-electron contribution dominant. It must be noted that the intraband and interband terms are wavelength dependent, so the enhancement of the non-linear optical properties due to particle size may hold at one wavelength, but not at another.

To summarise the results from MG theory, resonant enhancement of the non-linear optical properties occurs when the inclusions are metallic and the host dielectric. In addition, local-field effects may lead to enhancement of the overall nonlinearity, even off resonance in certain special cases.

\section{Bruggeman Theory}

The self-consistent Bruggeman approach (33) treats the composite as a random mixture of both metallic and insulating particles. The Bruggeman theory (also an effective medium theory) was first proposed by Bruggeman, and later analysed by Landauer (34) and Stroud (35). Alternative derivations have been devised by Aspnes (36). If the approximation of a spherical grain surrounded by a material of uniform dielectric constant, given by that of the effective medium, is made, the following can be derived: 


$$
0=f_{1} \frac{\boldsymbol{\varepsilon}_{1}-\boldsymbol{\varepsilon}_{\text {effective }}}{\boldsymbol{\varepsilon}_{1}+2 \boldsymbol{E}_{\text {effective }}}+f_{2} \frac{\boldsymbol{\varepsilon}_{2}-\boldsymbol{\varepsilon}_{\text {effective }}}{\boldsymbol{\varepsilon}_{2}+2 \boldsymbol{\varepsilon}_{\text {effective }}}
$$

It can be seen that the formula is symmetric with respect to the constituents, and the theory will be applicable for materials in which all components occupy large volume fractions. There is an important prediction by this theory. As the volume fraction of the particles increase, at some point they will begin to form continuous threads throughout the composite. At this percolation threshold, the properties of the composite may change significantly. The Bruggeman theory is more useful for its qualitative predictions of the functional dependence of the linear effective dielectric constant on the fill fraction in composites, than quantitative analysis.

At low volume fractions of metal particles, the Bruggeman and MG models are the same (37). As the volume fraction increases, the MG model predicts a sharp resonance, whereas the Bruggeman model predicts a broadening and weakening of the resonance. Sheng (38) modified and combined the two models, comparing the modified theory with experimental data for gold/silica composites favorably. His approach was to consider a particle with a core of one material with a shell of the other, with dimensions such that the volume fraction of each material is the same as that in the composite as a whole. The analysis then proceeds in the Bruggeman formalism.

Predictions of the non-linear properties of Bruggeman geometries are difficult, and strong simplifying assumptions have to be made. While there is good agreement in the linear limit, there is poor agreement between numerical simulations and theory for the nonlinear properties.

Genzel et al. (39) proposed a simple model for the dielectric function of small metal particles. This dielectric function was used to examine quantum size effects in the optical absorption spectra. They showed that for small particles of uniform size and shape, the SPR should shift and broaden, and show fine structure corresponding to transitions between discrete conduction band energy levels.

Gao and Li (40) studied the temperature dependence of the effective non-linear properties of metal/dielectrics composites on the basis of the decoupling approximation and the spectral representation. Numerical results with $\mathrm{Ag}: \mathrm{MgF}_{2}$ showed that the effective non-linear optical properties can vary greatly with temperature.

Hache et al. (41) have reported a self-consistent calculation of the non-linear response of the electrons of a small metal spherical particle. The model was applied numerically to the case of homogenous response of the electrons and it was shown that screening effects may be important in the non-linear response of spheres with small dimensions (few nanometers).

\section{The Fabrication of Gold Nanoparticles in Glasses}

There are a number of methods that are used to embed nanoparticles in various matrices. In this report, only those methods that have been used for Au nanoparticles are mentioned. In many cases, such as the sol-gel technique, there are many variations and preparations that are used, too many to be covered here. The interested reader is directed to the individual preparation methods referred to in the literature. Out of all the methods, the sol-gel method is the easiest method to prepare gold nanoparticles in glasses. There is no need for complicated equipment, and many of the reactions take place at low temperatures.

\section{Mechanical Methods}

Conventional melting of glasses with nanoparticles is probably the simplest method of embedding nanoparticles in a glass matrix. The process has been employed as far back as the Roman times (42). Gold nanoparticles implanted in glasses using conventional melting have been done by Hache et al. (43) and by Sasai and Hirao (44). A disadvantage of the technique is that low nanoparticle concentrations are obtained, due to low solubility and vaporisation.

Sputtering is another mechanical method of producing nanoparticle composites. Using radio frequency ( $\mathrm{rf}$ ) sputtering (45) and variants of the technique, various composite films can be made, in various configurations. Composites of $\mathrm{Au}: \mathrm{TiO}_{2}$ films have been prepared by a multitarget sputtering method (46) or by using rf-sputtering (47). Different matrices have also been produced using this technique. Au: $\mathrm{SiO}_{2}$ films were prepared (48) by co-sputtering $\mathrm{Au}$ and $\mathrm{SiO}_{2}$ in a multi-target magnetron sputtering system. Greater nanoparticle concentrations can be obtained using the sputtering technique, compared to conventional melting. Another variant of the sputtering technique is to use laser vaporisation to co-deposit $\mathrm{Au}$ with an $\mathrm{Al}_{2} \mathrm{O}_{3}$ dielectric vapour (49) in a low-energy cluster-beam deposition (LECBD) $(50,51,52)$ process. Cotell et al. (53) have used evaporation and ion beam assisted deposition (IBAD) to deposit Au clusters (5-30 nm) in $\mathrm{Nb}_{2} \mathrm{O}_{5}$. IBAD has advantages over ion-implantation, in that thicker thin films can be produced, and the films have higher density and contain fewer radiation-induced defects than ion-implanted films. Ion beam bombardment conditions or annealing temperature can change nanocluster size over a narrow range.

Ion-implantation is the most versatile of the mechanical methods to implant nanoparticles into glasses, and has been used to implant $\mathrm{Ag}$ and $\mathrm{Au}$ in silicate glasses (7). There is a high degree of control, so that thin films in waveguide configurations can be formed. High volume fractions can be obtained with well-defined vertical and horizontal 
dimensions. There are many factors that determine the growth and distribution of the nanoparticles, one of the more important being the composition of the matrix. The formation and size of the implanted nanoparticles are highly dependent on the composition (54). For example, the total dose, current density and substrate temperature can control the particle size $(55,56)$. When the fluence (the amount of ions incident on the sample) of the ions is increased, and the acceleration decreased, the formation and growth of the nanoparticles is increased (57). The depth of the nanoparticles in the thin film can be controlled by the implantation energy. Post-implantation processes can also be used to control the size and size-distribution of the implanted particles (58). Heat treatments subsequent to ion-implantation causes the growth of $\mathrm{Au}$ nanoparticles $(57,59,60)$ and increases the volume fraction (61). The particles grow mainly through the diffusion-controlled Ostwald ripening mechanism: within a system of dispersed particles of varying size in a medium in which they have some solubility, the smaller particles dissolve and the larger ones grow $(62,63)$. The growth is controlled by diffusion of Au in the silica matrix.

One disadvantage of ion implantation is that there are two mechanisms of optical absorption in nanoparticle/matrix composites. The first is absorption due to defects and highly strained sites created during the implantation process, due to the high energies. In silica, these defects result in broad absorption bands at 5, 5.8, 7.5 and $7.6 \mathrm{eV}(64,65,61)$, but these are dominated by the second mechanism, which is the interaction of the metal particles with the incident radiation.

\section{Chemical Methods}

The chemical methods used to prepare nanoparticle composites can be broadly divided into two methods, electrochemical deposition and the sol-gel method. The solgel method is a mature field. It has been used to deposit many species of nanoparticles into various sols, and there are many reports detailing this method. The large number of methods and their variants are too many to report here, so just the important ones are mentioned.

Au nanoparticles have been deposited in nanoporous alumina membranes $(66,67,68)$, and the technique that has been used is electrochemical deposition. The details of this technique were described by Hornyak et al. (69).

Sol-gel processing has been used to produce $\mathrm{Au}$ nanoparticles in glasses (70). The methods that can be used are varied, such as the inverse micell/sol-gel method (71), and they can be complicated. However, there is great freedom in the matrices that can be used. Au nanoparticles have been incorporated in $\mathrm{BaTiO}_{3}(72)$, in $\mathrm{Al}_{2} \mathrm{O}_{3}(73,74,75)$ and silica (76). Epifani et al. (77) have reported a method of preparing $\mathrm{Ag}$ and $\mathrm{Au}$ nanoparticles in $\mathrm{SiO}_{2}, \mathrm{TiO}_{2}$, and $\mathrm{ZrO}_{2}$ thin films, using the sol-gel process.

\section{Techniques Used to Characterise Nanoparticle Composites}

The average size of the nanoparticles in the matrix is the first property that has to be established. The most straightforward method is to examine the structure in a transmission electron microscope (TEM), where the shape, size and structure (78) of the nanoparticles can be visually inspected. It is even possible to determine if alloy phase formation occurs in the nanoparticles (79). Another technique is to use X-ray diffraction and Scherer's equation (80). The full width at the half maximum (FWHM) of the peaks in the XRD spectrum is related to the average grain size $(R)$ by:

$$
\mathrm{FWHM}=0.94 \lambda / R \cos \theta \text {, }
$$

where full-width at half-maximum (FWHM) and $\theta$ (the diffraction angle) are in radians, and $\lambda$ and $R$ are in $n m$ units. Another method to calculate the average radius of gold nanoparticles $r$ is by using the following (43):

$$
r=v_{f} \mid \Delta W_{1 / 2} \text {, }
$$

where $v_{\mathrm{f}}$ is the Fermi velocity of gold and $\Delta W_{1 / 2}$ is the FWHM of the absorption band due to the plasmon resonance of gold particles.

Degenerate four-wave mixing (81) (DFWM) is used to determine the third-order non-linear susceptibility $\chi^{(3)}$. There are many flavours of this technique, but the basic setup is as follows. Two strong pump (or write) beams are incident on the medium and a weaker probe (or read) beam examines these beams. When the three beams are incident on a non-linear optical medium, they generate a fourth beam, the phase conjugate, which is counter-propagating to the probe. The magnitude of the $\chi^{(3)}$ is estimated from a measurement of the intensity of the phase conjugate beam relative to that of a reference sample.

DFWM measures the magnitude of $\chi^{(3)}$. Its sign is determined by the Z-scan technique (82), which is as follows: a collimated beam is focused into the sample, and the sample is then translated along the optical ( $Z$ ) axis so that it goes from one side of the focus to the other. The change in refractive index due to third-order nonlinearity causes an intensity dependent lensing effect in the medium, leading to a focusing or defocusing of the beam (which can be measured by an aperture detector). The behaviour of the transmitted intensity is different for materials with positive or negative third-order nonlinearity.

Smith et al. (83) has shown how to directly measure the non-linear absorption coefficient on a gold film using the z-scan technique. He also reported a method to perform and analyse data obtained from the z-scan technique.

Quinten (84) has described a method of determining the dielectric constant, which is based on a Kramers-Kronig relation for the extinction. A generalised Newton-Raphson iteration method is applied to the measured extinction and 
the Kramers-Kronig data, and the dielectric constant obtained.

\section{The Optical Properties of Gold Nanoparticles in Glasses}

There are a number of studies on the optical properties of gold, too many to mention here. However, Thèye presented a thorough study of thin gold films (85), for a range of films less than $21 \mathrm{~nm}$ thick, in the wavelength range $200 \mathrm{~nm}$ to 2000 nm. Johnson and Christy (86) measured the optical properties of the noble metals in the range $\sim 200 \mathrm{~nm}$ to $\sim 2500 \mathrm{~nm}$ as well. Barker (87) demonstrated that the surface plasmon technique could be used to determine the optical properties of gold. Weber and McCarthy (88) used the technique, but only at two wavelengths. Berthier and Peiro (89) measured the optical absorption of gold granular films, and concluded that the IR absorption could be attributed to classical surface plasmon modes. The use of the surface plasmon resonance technique on gold is difficult, as the surface plasmon resonance becomes a very broad feature in the blue/green part of the spectrum due to the d-band electrons. Use of large-angle scans are therefore necessary, but when these problems are overcome, the technique is a simple, quick and accurate method for the determination of the real and imaginary dielectric constant of gold (from the ultraviolet to the near infrared).

Innes and Sambles (90) have determined the real and imaginary parts of the dielectric constant of gold at various wavelengths using the surface plasmon-polariton technique between $400 \mathrm{~nm}$ and $800 \mathrm{~nm}$. They compared the results to other work and found good agreement. They related their data to that of free-electron theory, and found close confirmation for wavelengths above $700 \mathrm{~nm}$. Papadogiannis et al. (91) have investigated the second and third harmonic generation efficiencies of gold in a broad intensity regime up to the plasma threshold.

The optical properties (linear and non-linear) of nanoparticles in a matrix are mainly dependent on the properties of the nanoparticles, the matrix in which the nanoparticles are embedded, and the interaction between the nanoparticles and the matrix. In addition, there are other factors that have to be taken into consideration. An important property that must be taken into account is the time-dependent dynamics of the processes (32). Response and relaxation times occur in the order of picoseconds and femtoseconds (the response time for $\mathrm{Au}$ in glasses was measured as less than 5 ps (19,32)), implying the nonlinearity is electronic in origin. The various processes appear on different time-scales as well. This explains the differences in the reported values of the susceptibility of Au nanoparticles - earlier investigations used laser pumps with long pulse-widths, resulting in a number of processes, all contributing to the measured value. Liao et al. (92) found that in $\mathrm{Au}: \mathrm{TiO}_{2}$, the response time was extremely fast and highly dependent on Au concentration. The non-linear optical contribution was in three parts: instantaneous response from interband electric-dipole transitions between the $d$-valence and $s-p$ conductions bands $\left(\sim 10^{-13} s\right)$; hot electron excitation close to the SPR (several picoseconds); and thermal contribution (nanoseconds) that increased slightly with Au concentration. One can get a larger $\chi^{(3)}$ if a picosecond laser probes the composite, as hot electron contribution can then be fully utilized. Before 1998, all optical measurements were done with pulse widths of several picoseconds to several nanoseconds - on this time scale, $\chi^{(3)}$ (the third-order non-linear susceptibility of the metal nanoparticles) is dominated by so-called hot-electron excitation. Liao et al. (93) measured $\chi^{(3)}$ at various pulse durations, and at 200fs, $\chi^{(3)}$ had a maximum of $\sim 1.7 \times 10^{-7}$ esu, $\sim 25$ times smaller than with pulse duration of $70 \mathrm{ps}$. The trend of $\chi^{(3)}$ on the Au concentration was almost the same for different pulse durations. $\chi^{(3)}$ depends strongly on the pulse width of the probe laser. The optical linearity in $\mathrm{Au}: \mathrm{SiO}_{2}$ takes a certain time to respond to the probe laser, and a pulse width longer than femtoseconds is necessary to capture large $\chi^{(3)} \cdot \chi_{\mathrm{m}}{ }^{(3)}$ of $\sim 4.2 \times 10^{-8}$ and $3.4 \times 10^{-9}$ esu were measured on time scales of 70 ps and 200 fs respectively. Liao et al. (93) suggested that the hot-electrons at the pulse width of $200 \mathrm{fs}$ only partially contribute to $\chi^{(3)}$. When a femtosecond pulse is used, the contributions from both interband and hot-electron excitation should be equally considered. When a picosecond pulse is used, the thermal effect should be included. When a nanosecond pulse is used (as in earlier experiments), the thermal effect becomes dominant.

Puech et al. (94) measured the phase relaxation time and the non-linear susceptibility of gold nanoparticles in colloids. They found that for particles with size between 5-40 nm, the phase relaxation time was of the order of, or faster than, $20 \mathrm{fs}$. They also measured the susceptibility of the particles as $\sim 2 \times 10^{-16} \mathrm{~m}^{2} \mathrm{~V}^{-2}$. Ahmadi et al. (95) measured the picosecond dynamics of gold nanoparticles in colloids. Their particle size was greater than $8 \mathrm{~nm}$ (below that was found to experience quantum confinement (96)). They found two time constants, one of 2.5 ps and a slower one of $>50$ ps. They interpreted their observations as the rapid formation of a hot-electron distribution within the particles on a time scale shorter than their time resolution. The equilibration of the hot-electrons occurred in accord with the two temperature model (97) (separating the heat capacity of metals into lattice and electronic parts) via an electronelectron relaxation process of $2.5 \mathrm{ps}$, followed by an electronphonon process of $>50$ ps. Cotell et al. (53) measured gold 
nanoparticles in $\mathrm{Nb}_{2} \mathrm{O}_{5}$, and found that the relaxation was fast, on the order of several picoseconds, and was also dependent on the excitation intensity. He also noted that $\left|\chi^{(3)} x x x x\right|$ (the magnitude of the third order susceptibility tensor element) was eight times larger than that reported for Au-doped glasses. They proposed a number of reasons for this, but the most probable would be that the refractive index of the oxidised niobium was larger, resulting in larger local field factors. Perner et al. (98) studied the damping of the SP in gold nanoparticles. They found a SP line broadening which builds up within $\sim 1$ ps after excitation. They suggested that the line broadening was by far the dominant optical nonlinearity of the system. Hodak et al. (99) found that the characteristic time-scale for electron-phonon coupling did not change with particle size for Au (they looked at Au nanoparticles in aqueous solution). This suggested that phonon emission inside the particle dominates the dynamics. These considerations are important for designing devices with fast response times, which are tunable through changing the size or composition of the nanoparticles. The non-linear response of glasses containing metal nanoparticles is dominated by two relaxation processes (100): (1) a fast relaxation process due to electron-phonon coupling which leads to thermal equilibrium between the electron and the lattice in the metal nanoparticle system and (2) slow relaxation process due to thermal diffusion of the excess heat from metal nanoparticles to the glass matrix.

Link and El-Sayed (101) have published a review of the spectral and relaxation dynamics of $\mathrm{Au}$ nanoparticles in colloidal solution. The interested reader is directed to the comprehensive commentary of the relaxation dynamics of the surface plasmon oscillation.

The plasmon peak wavelength depends on the dielectric constants of the matrix glass and the particles. Fukumi et al. (60) showed that in Au ion implanted silica glass samples, the total volume of $\mathrm{Au}$ particles did not vary with heating conditions. Therefore, there was no change in the composition and the dielectric constant of the matrix glass. The wavelength shift was then due to the size dependence of the particles' dielectric constant. They demonstrated theoretically that $\chi^{(3)}$ was proportional to the volume fraction of the Au colloid particles, the $\chi_{m}{ }^{(3)}$ (of the metal particles on their own) of the particles and to the fourth power of the particle radius. They also showed that $\chi_{\mathrm{m}}{ }^{(3)}$ had little dependence on the particle radius. The dependence of $\chi^{(3)}$ on the absorption properties and the radius and concentration of the Au particles is mainly attributable to the particle size dependence of the imaginary part of the dielectric constant of the Au particles.

The third-order non-linear susceptibility $\chi^{(3)}$ depends on the nanoparticle size, the matrix, the resonant wavelength, the laser pulse width and the volume fraction. Values of $\chi^{(3)}$ for $\mathrm{Au}: \mathrm{Al}_{2} \mathrm{O}_{3}$ (particles 2-10 nm in size) have been measured of the order of $10^{-8}$ esu (102). In glasses, $\chi^{(3)}$ has been reported of the order of $10^{-12}-10^{-11}$ esu (particles less than $30 \mathrm{~nm}$ ) (19), and $1.2 \times 10^{-7}$ esu (103). Hosoya et al. (75) reported $\chi_{\mathrm{m}}{ }^{(3)}$ (susceptibility of the metal nanoparticles) of $\mathrm{Au}: \mathrm{Al}_{2} \mathrm{O}_{3}$ in the range $0.5-2.3 \times 10^{-5}$ esu. Mennig et al. (104) reported $\chi_{\mathrm{m}}{ }^{(3)}$ to be $2.6 \times 10^{-6}$ esu in $\mathrm{Au}: \mathrm{SiO}_{2}$. Hosoya et al. (75) suggested the simultaneous formation of the alumina network and $\mathrm{Au}$ particle prevented the doped alumina from reaching thermodynamic equilibrium. The electron transfer of the Au particle to the surrounding matrix affected the electronic properties of the doped film. This caused an enhancement of $\chi_{m}{ }^{(3)}$. The energy relaxation time was obtained between 1-3 ps. When the figure of merit $\left(\chi^{(3)} / \alpha \tau\right)$ is compared with $\mathrm{Au}: \mathrm{SiO}_{2}$ $\left(\sim 2\right.$ esu.cm. $\left.s^{-1}\right), \mathrm{Au}: \mathrm{Al}_{2} \mathrm{O}_{3}$ is significantly greater ( $\sim 50$ esu.cm. $\left.s^{-1}\right)$.

The non-linear susceptibility is also different for manufacturing methods. For Au nanoparticles in melt glass, reported values are $10^{-12}$ esu $(43,32)$, while for ion-implanted composites, values were measured as $1.0 \times 10^{-10}$ esu. In this case, the increase in $\chi^{(3)}$ is primarily due to the high local density of $\mathrm{Au}$ nanoparticles. The high Au nanoparticle concentrations were attributed to the $\chi^{(3)}$ values of $10^{-7}$ esu for sputtering-derived $\mathrm{SiO}_{2}$ (105) and ion-implanted $\mathrm{SiO}_{2}$ glass (103). For sol-gel derived $\mathrm{SiO}_{2}$, $\chi^{(3)}$ was $8 \times 10^{-9}$ esu (106). Lee et al. (107) measured $\chi^{(3)}$ with a maximum value of $2.3 \times 10^{-8}$ esu in $\mathrm{Au}^{\mathrm{S}} \mathrm{SiO}_{2}$ thin films with $0.73 \%$ $\mathrm{Au}$ concentration, produced by sol-gel processing. Values ranged $0.4-2.3 \times 10^{-8}$ esu in the range $530-570 \mathrm{~nm}$, higher than the $7.7 \times 10^{-9}$ esu reported by Matsuoka et al. (108)

Ostuki et al. (72) showed that the surface plasmon resonance shifted to longer wavelengths, and the peak intensity decreased with increasing heat-treatment temperatures (increasing particle size), for gold particles in $\mathrm{BaTiO}_{3}$. They also showed that the $\chi^{(3)}$ of $\mathrm{Au}: \mathrm{BaTiO}_{3}$ was $\sim 10$ times that of $\mathrm{Au}: \mathrm{SiO}_{2}$, measured by Kineri (109). The large values could be attributed to the $\mathrm{BaTiO}_{3}$ matrix.

The dielectric matrix influences the wavelength at which there is surface plasmon absorption. It was found (110) that when $\mathrm{Au}$ nanoparticles were incorporated in a $\mathrm{TiO}_{2} / \mathrm{SiO}_{2}$ complex oxide thin film, the wavelength of the maximum absorption varied linearly from $540 \mathrm{~nm}$ to $615 \mathrm{~nm}$ with molar ratio of $\mathrm{TiO}_{2}$. In the limit of low concentration, $\chi^{(3)}$ is proportional to the fourth power of the local field factor $f$. The larger the refractive index of the matrix, the larger the $f$ of the composite. Liao et al. (92) showed that $\chi^{(3)}$ can be enhanced by using a matrix with a larger refractive index.

Part II will be published in the next issue, which will include all the references 